\title{
Photoabsorption and Photoionization Studies of the Amino Acid Proline in the VUV Region
}

\author{
L. H. Coutinho ${ }^{b}$, M. G. P. Homem ${ }^{b}$, R. L. Cavasso-Filho , R. R. T. \\ Marinho $\sharp$, A. F. Lago ${ }^{\natural *}$, G. G. B. de Souza ${ }^{\natural}$, and A. Naves de Brito ${ }^{\natural \sharp}$ \\ ${ }^{b}$ Laboratório Nacional de Luz Síncrotron (LNLS), Box 6192, 13084-971, Campinas-SP Brazil \\ \# Instituto de Física, Universidade de Brasília, Box 4455, 70910-900, Brasília-DF Brazil \\ ${ }^{\natural}$ Instituto de Química, Universidade Federal do Rio de Janeiro, \\ Cidade Universitária, Ilha do Fundão, 21949-900, Rio de Janeiro-RJ Brazil
}

Received on 11 January, 2005

\begin{abstract}
Ionic fragmentation of the sublimated amino acid DL-proline has been studied using time-of-flight mass spectrometry and synchrotron radiation. Total ion yield and mass spectra were recorded in the 13 to $21.6 \mathrm{eV}$ energy range. Partial ion yields have been calculated for the produced fragments and the results analyzed in a comparative way. Mass spectrum of proline previously obtained at $21.21 \mathrm{eV}$ using photons from a discharge lamp (He I), was used as reference in the comparison to the synchrotron radiation based spectra. The loss of the $\mathrm{COOH}$ fragment represents the most probable dissociation pathway following the photoionization of DLproline in the valence region. These are the first results of total and partial ion yields spectra for this molecule in its gas phase in the valence region using time-of-flight spectrometry.
\end{abstract}

\section{INTRODUCTION}

In recent years, there has been an increasing interest in the spectroscopic study of amino acids due to the fundamental importance of these molecules, which are building blocks of proteins. Knowledge on the valence shell electronic structure of amino acids is necessary for understanding the details of their photo-interaction and reactivity. In addition, valence photoionization studies provide a means for understanding the photo-stability, as well as the pathways leading to the photofragmentation products of these molecules following Vacuum Ultraviolet (VUV) excitation or ionization. Such information is of great importance for several areas, for instance, astrophysics and photochemistry, since amino acids have been found in the interstellar medium and meteoritic materials [1-3].

Only a few papers devoted to the experimental study of the amino acids in the VUV regions have been found in the literature. Powis et al. [4] reported on the valence and C 1s core level photoelectron spectra and quantum calculations of gaseous alanine and threonine. Jochims et al. [5] reported an extensive photoionization study in the $6-22 \mathrm{eV}$ energy range, using quadrupole mass spectrometry, of five amino acids: glycine, $\alpha$-alanine, $\beta$-alanine, $\alpha$-aminoisobutyric acid and $\alpha$ valine. The Time-Of-Flight (TOF) spectra of the sublimated alpha amino acids L-alanine, L-proline, L-valine and glycine, have been recently reported by Lago et al. [6], from photodissociation induced by $\mathrm{He} \mathrm{I}(21.21 \mathrm{eV})$ photons. This previous results for proline [6], using a He I lamp and a similar spectrometer to the one used in this work, have also been used here for sake of comparison.

*Present address: Department of Chemistry, University of North Carolina, Chapel Hill, NC, 27599-3290, USA
In this paper we report the dissociative photoionization of the amino acid DL-proline in the gas phase, by using Time-Of-Flight Mass Spectrometry (TOF-MS). Photons from a synchrotron radiation source and a discharge lamp (He I) were used as ionizing agents. The resulting photoionization products as well as fragmentation pathways leading to those species have been presented and discussed. Total Ion Yield (TIY) and Partial Ion Yield (PIY) spectra have been recorded in the 13 - $21.6 \mathrm{eV}$ energy range. Mass spectra have been also recorded as a function of photon energy. The resulting decay channels were analyzed in a comparative way. The spectra are, in general, similar to those obtained from electron impact $(70 \mathrm{eV})$ experiments [7]. Within our knowledge, no similar TOF-MS data is available in the literature.

\section{EXPERIMENT}

The experimental set-up used has been described in detail in Ref. [8]. The ions produced by photoionization and photodissociation were detected using a TOF spectrometer schematically shown in Fig. 1. The mass spectra are recorded by the PhotoElectron PhotoIon COincidence technique (PEPICO). TIY and PIY spectra were obtained by scanning the photon energy and recording the corresponding ions produced in the photoionization process. In the PEPICO spectra, the relative areas of the peaks reflect the relative abundance of each species and the width is proportional to the Kinetic Energy Release (KER) during the photofragmentation process. The TOF spectrometer was mounted on a chamber that can rotate with respect to the polarization vector of the exciting beam, and was kept in the pseudo magic angle of $54.7^{\circ}$ during the measurements. At this angle, possible angular effects (discrimination) due the angular distribution of the photoelectron are avoided since this distribution is independent of the asymmetry parameter beta $[8,9]$. 


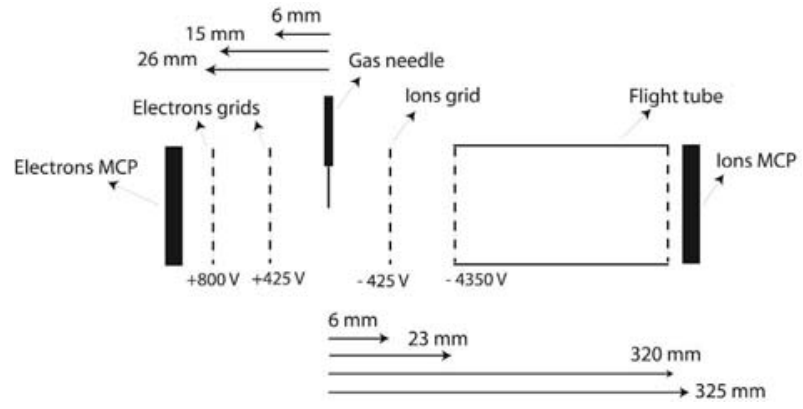

FIG. 1: Schematic drawing of the TOF mass spectrometer used for the measurements. The sublimated amino acid enters the ionization region by a needle placed between two plates that accelerate the ions to the flight tube and MicroChannel Plates (MCP) detectors on the right side, and the electrons to the MCP detectors on the left side. The arrival of an electron in the detector initiates a clock that marks the flight-time of the correlated ion.

The vacuum chamber base pressure is usually maintained at about $10^{-9}$ mbar. The photons were provided by the Toroidal Grating Monochromator beamline (TGM) [10] from the LNLS storage ring, located in Campinas-SP, Brazil. This beamline provides linearly polarized radiation from a bending magnet and operates in the range from 12 to $300 \mathrm{eV}$. The photon intensity was recorded by means of a light sensitive diode. In order to minimize contributions from higher order harmonics in the photon beam, a mixed metallic/gas filter has been added to the beamline. It basically consists in filling a certain region of the beamline with a rare gas (neon), which is isolated from the rest of the vacuum system by two thin metallic foils [11]. Therefore, contributions from higher-order light from the monochromator were negligible in the energy range studied. When using this filter we are able to perform measurements at energies up to $21.6 \mathrm{eV}$, the absorption edge of Ne. The DL-proline sample was purchased from SigmaAldrich in the form of crystalline powder with minimum purity of $99 \%$, and was used without any further purification. Due to the low vapor pressure of this compound, it was necessary to use a heated source for sublimation. More details on the sublimation procedure are available in Ref. [6]. The pressure in the chamber was kept in the $10^{-7}$ mbar range during the experiments. In order to confirm the absence of decomposition of the samples upon heating, we have performed a Liquid Chromatographic-Mass Spectrometry (LC-MS) experiment after the sublimation procedure. The LC-MS results confirmed that no degradation occurred before sublimation of the sample in the temperature range used during our measurements.

\section{RESULTS}

We recorded several PEPICO spectra using synchrotron radiation, to analyse both total and partial ion yields. The spectral range from 13 to $22 \mathrm{eV}$ was covered, with points being

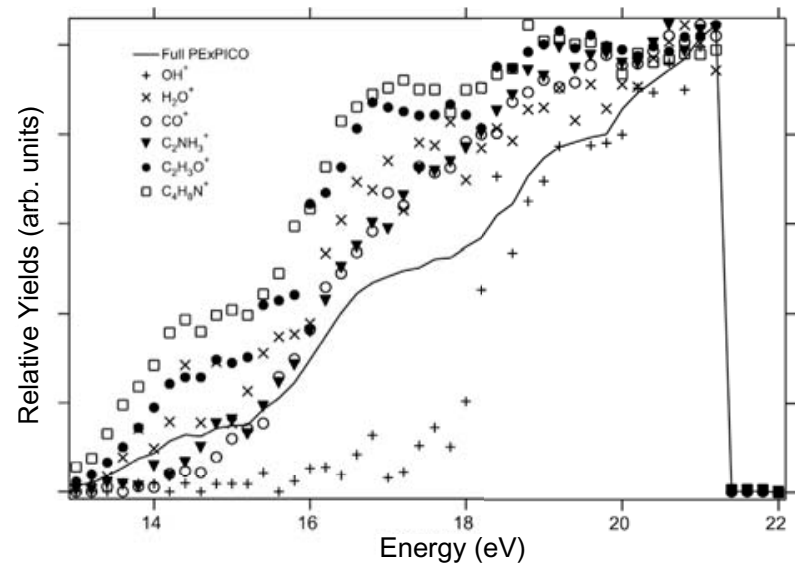

FIG. 2: Total ion yield and partial ion yield of the produced fragments after photoionization of DL-proline. Above $21.6 \mathrm{eV}$ the intensities drop due to the effect of the harmonic filter.

collected every $0.2 \mathrm{eV}$. From this set of spectra we were able to construct the graph shown in Fig. 2, where for each photon energy we plot the relative production of the main fragments (PIY) and the TIY. The yields are normalized at their maximum values. The pressure in the experimental chamber and the intensity of the photon beam were used to normalize the data. From these curves we can see that the production of ions increases as the photon energy increases. Besides, a higher slope between 16 and $18 \mathrm{eV}$ can also be observed. From the TIY curve we can distinguish three structures around the energies $14.4 \mathrm{eV}, 17.0 \mathrm{eV}$ and $19.2 \mathrm{eV}$. Above $21.6 \mathrm{eV}$ the intensities drop due to the effect of the harmonic filter. The values of the PIY obtained for DL-proline as function of the photon energy are also shown in table 1. It is important to compare both Fig. 2 and table 1 when analyzing the results. As an example let us take $\mathrm{OH}^{+}$: in the graph it shows a large increase on its production above $18 \mathrm{eV}$, but if we look on the table we observe that even at $21.21 \mathrm{eV}$ its production is only about $1 \%$ of total. The PIY curves on the graph should not be quantitatively compared one to the other, since their intensities have been normalized at their maximum values, and the table should be used to have a better understanding of the results.

To complement the results above we took PEPICO spectra on the top of the main features observed in the TIY spectra, and the results are presented in Figs. 3 and 4. From table 1 the main fragments resulting from the photofragmentation of DL-proline are $\mathrm{C}_{4} \mathrm{H}_{8} \mathrm{~N}^{+}$and $\mathrm{C}_{2} \mathrm{H}_{3} \mathrm{O}^{+}$, which correspond to approximately $90 \%$ of all detected fragments in the lower part of the spectra, decreasing to about $60 \%$ at $21.21 \mathrm{eV}$. The parent ion presented a very low intensity, suggesting an efficient molecular bond rupture and instability of this ion in the time scale of the experiment (the flight-time of this ion in our TOF spectrometer was approximately $4.2 \mu \mathrm{s}$ ). The most intense peak in all the PEPICO spectra shown in Figs. 3 and 4 is attributed to $\mathrm{C}_{4} \mathrm{H}_{8} \mathrm{~N}^{+}$, which corresponds to the loss of a neutral fragment $\mathrm{COOH}$, which is in agreement with results previously obtained for L-proline by the He I photoionization [6]. 


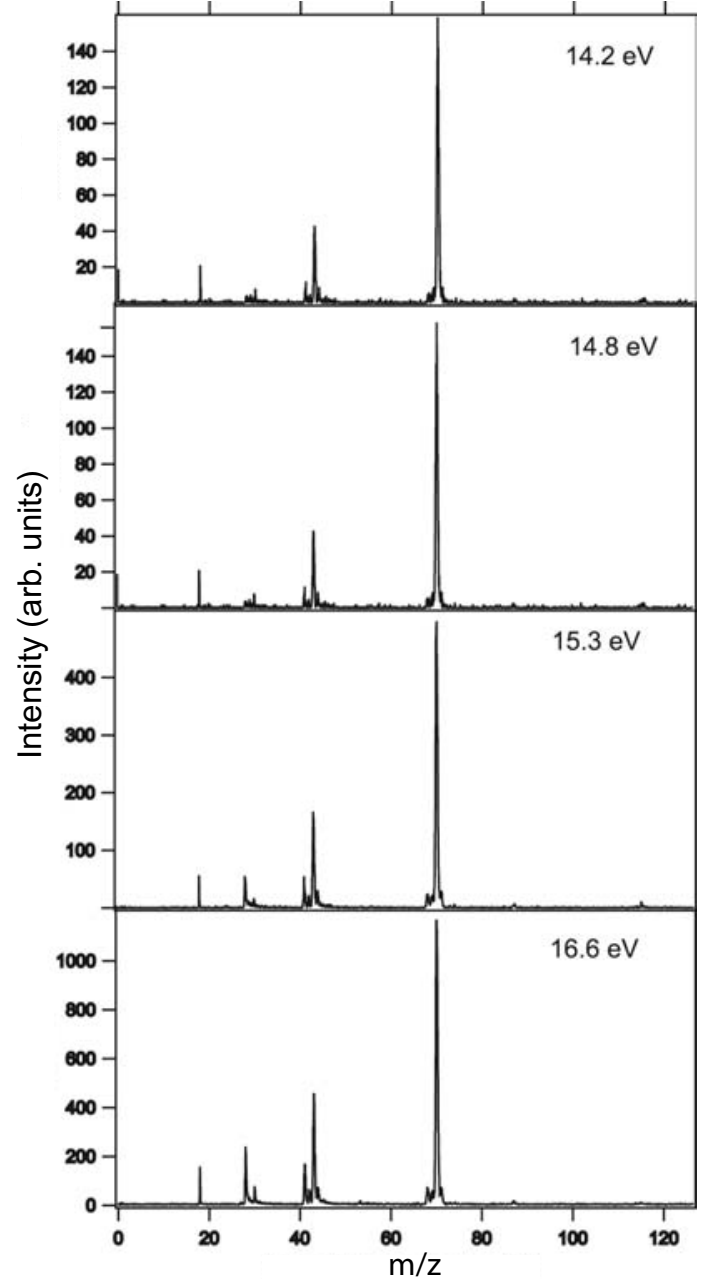

FIG. 3: Mass spectra of DL-proline at 14.2, 14.8, 15.3 and $16.6 \mathrm{eV}$ photon energies.

We also point out the presence of ions formed from rearrangement reactions, such as $\mathrm{OH}^{+}, \mathrm{H}_{2} \mathrm{O}^{+}$and $\mathrm{CO}^{+}$. However, the presence of water contamination in the peaks at $\mathrm{m} / \mathrm{z}=17$ and 18 can not be discarded. The formation of fragments such as $\mathrm{OH}^{+}$and $\mathrm{CO}^{+}$may result from the fast dissociation of the $\mathrm{COOH}^{+}$ion, which was absent from the mass spectra. The $\mathrm{CO}^{+}$fragment presents a dramatic increase in its production with the increasing of the photon energy when compared to the other fragments, as can be observed both from the table 1 and from Figs. 3 and 4.

The spectrum of L-proline at $21.21 \mathrm{eV}$ (He I) shown in Fig. 5 was taken from Lago et al. [6]. In this figure it is possible to identify a higher number of ionic fragments than in our present results for DL-proline, taken with synchrotron radiation. Also the relative productions of the main fragments are different. One hypothesis to explain this divergence is the occurrence of mass discrimination in the detector used in our spectrometer. The efficiency detection of the microchannel plates is a function of the kinetic energy of the fragments [8]. In this sense, the heavier masses would have smaller detection

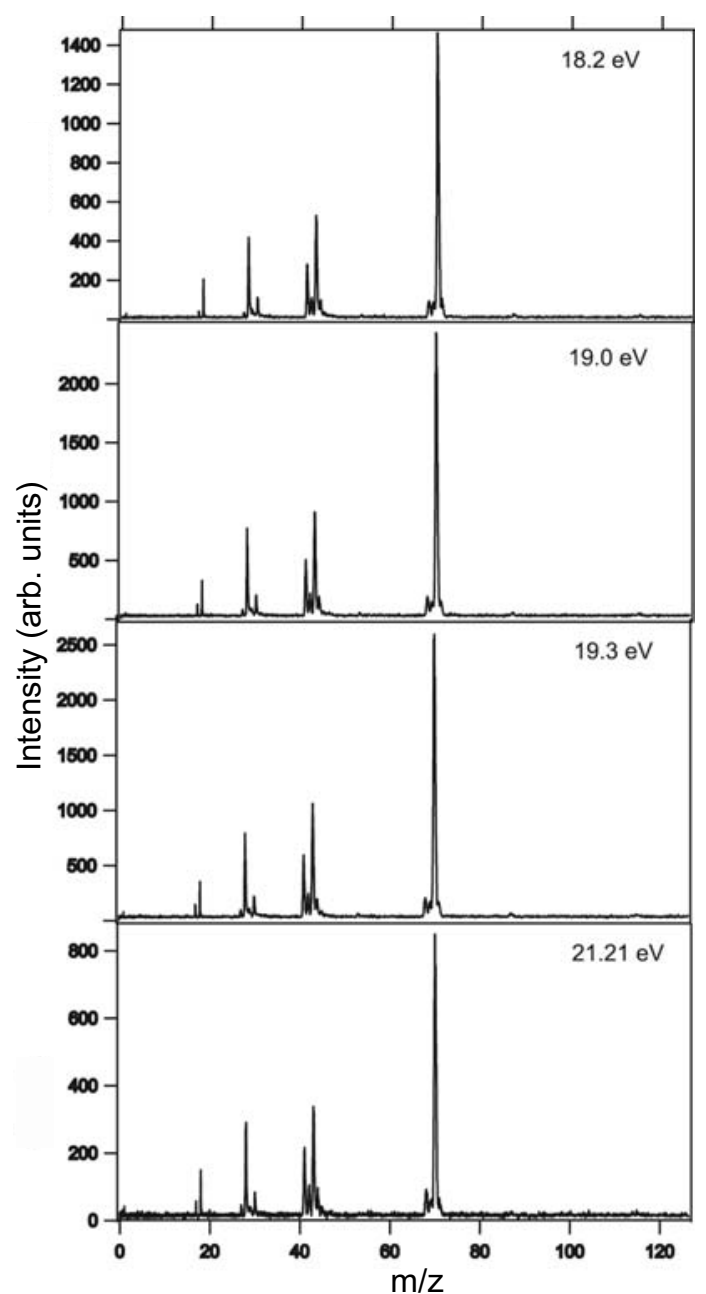

FIG. 4: Mass spectra of DL-proline at 18.2, 19.0, 19.3 and $21.21 \mathrm{eV}$ photon energies.

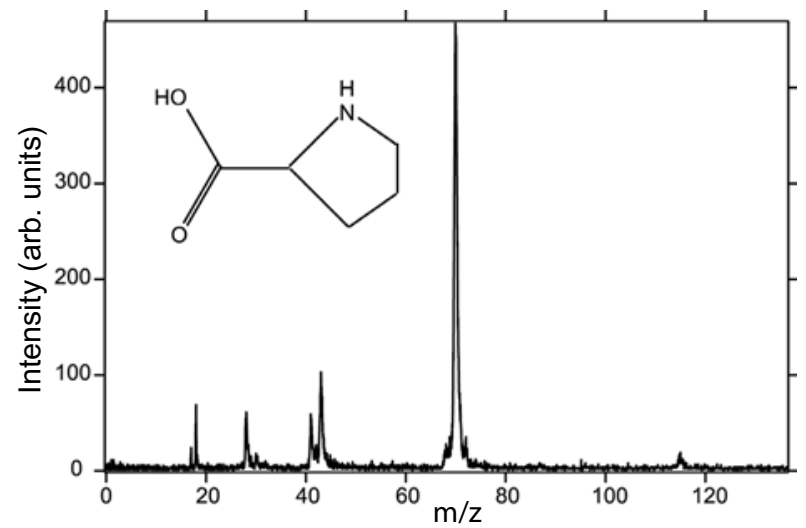

FIG. 5: Mass spectra of L-proline taken from Lago et al. [6] using a He I lamp. 
TABLE I: PIY in \% for the amino acid DL-proline after photoionization for several photon energies.

\begin{tabular}{|c|c|c|c|c|c|c|c|c|c|c|}
\hline \multirow{2}{*}{\multicolumn{2}{|c|}{$\overline{\overline{\text { Fragment } \mathrm{m} / \mathrm{z}}}$}} & \multicolumn{9}{|c|}{ Photon Energy $(\mathrm{eV})$} \\
\hline & & 14.2 & 14.8 & 15.3 & 16.6 & 18.2 & 19.0 & 19.3 & 20.2 & 21.2 \\
\hline$\overline{\mathrm{H}^{+}}$ & 1 & & & & & & & & 0.4 & 0.4 \\
\hline $\mathrm{OH}^{+}$ & 17 & & & & & 0.4 & 0.7 & 0.7 & 0.9 & 0.9 \\
\hline $\mathrm{H}_{2} \mathrm{O}^{+}$ & 18 & 3.1 & 8.9 & 2.5 & 2.7 & 2.7 & 2.3 & 2.2 & 2.4 & 2.8 \\
\hline $\mathrm{HCN}^{+}$ & 27 & & & & & & 0.4 & 0.4 & 0.9 & 1.0 \\
\hline $\mathrm{C}_{2} \mathrm{H}_{3}{ }^{+}$ & & & & & & & & & & \\
\hline $\mathrm{CO}^{+}$ & 28 & & & 5.3 & 9.1 & 10.5 & 10.9 & 10.7 & 10.3 & 10.7 \\
\hline $\begin{array}{l}\mathrm{CH}_{2} \mathrm{O}^{+} \\
\mathrm{NO}^{+}\end{array}$ & 30 & 1.6 & 1.5 & 1.2 & 2.3 & 2.2 & 2.5 & 2.4 & 2.9 & 2.8 \\
\hline $\begin{array}{l}\mathrm{C}_{2} \mathrm{NH}_{3}{ }^{+} \\
\mathrm{C}_{3} \mathrm{H}_{5}{ }^{+}\end{array}$ & 41 & 2.7 & 2.8 & 4.4 & 7.1 & 8.5 & 8.6 & 8.4 & 8.5 & 9.5 \\
\hline $\begin{array}{l}\mathrm{C}_{2} \mathrm{NH}_{4}^{+} \\
\mathrm{C}_{2} \mathrm{H}_{2} \mathrm{O}^{+}\end{array}$ & 42 & & & 1.8 & 2.4 & 3.5 & 3.5 & 3.0 & 3.2 & 4.1 \\
\hline $\mathrm{C}_{2} \mathrm{H}_{3} \mathrm{O}^{+}$ & 43 & 19.2 & 17.6 & 18.8 & 17.4 & 17.1 & 15.7 & 17.1 & 17.0 & \\
\hline $\mathrm{C}_{2} \mathrm{H}_{4} \mathrm{O}^{+}$ & 44 & 2.1 & 1.6 & 4.1 & 4.1 & 4.3 & 4.1 & 2.4 & 3.5 & 3.6 \\
\hline $\mathrm{CHO}_{2}+$ & 45 & & & 1.1 & & 0.9 & & & & \\
\hline $\mathrm{C}_{4} \mathrm{H}_{6} \mathrm{~N}^{+}$ & 68 & & & 2.5 & 1.7 & 2.8 & 2.7 & 3.9 & 3.2 & 3.1 \\
\hline $\mathrm{C}_{4} \mathrm{H}_{7} \mathrm{~N}^{+}$ & 69 & & & 2.0 & 1.0 & & 1.8 & 2.4 & 2.0 & 1.6 \\
\hline $\mathrm{C}_{4} \mathrm{H}_{8} \mathrm{~N}^{+}$ & 70 & 71.2 & 67.6 & 56.3 & 50.8 & 46.9 & 45.3 & 41.1 & 44.8 & 42.6 \\
\hline $\mathrm{C}_{2} \mathrm{HNO}_{2}{ }^{+}$ & -71 & & & & & & & 6.7 & & \\
\hline
\end{tabular}

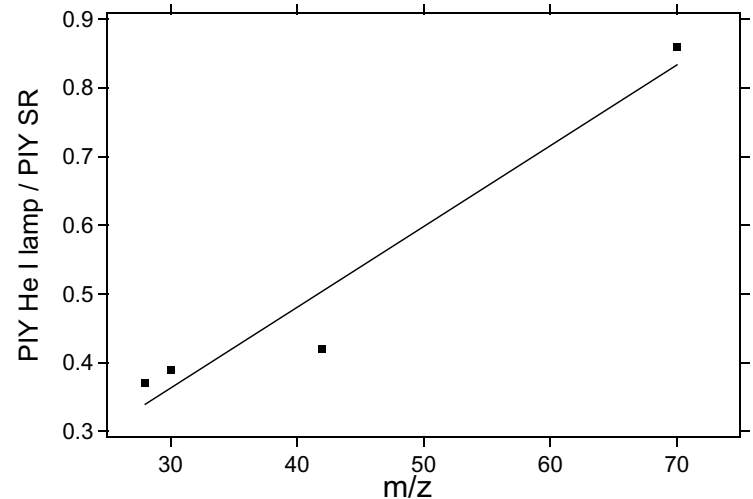

FIG. 6: The graph shows the ratio between the partial ion productions for the observed fragments using He I lamp and synchrotron radiation (SR) at $21.21 \mathrm{eV}$. The point at $\mathrm{m} / \mathrm{z}=42$ shows the contribution of the peaks at $\mathrm{m} / \mathrm{z}=41,42$ and 43 in the mass spectra in order to minimize the effects due the different mass resolution between the spectra obtained with He I and synchrotron radiation. The peaks at $\mathrm{m} / \mathrm{z}=17,18$ was not included due the different water contamination in spectra. efficiency. To try to confirm this hypothesis we constructed the graph in Fig. 6, where we compare the relative ionic productions for two spectra taken at $21.21 \mathrm{eV}$, one from Ref. [6], using the He I lamp, and the other using synchrotron radiation. Care was taken to add together all unresolved peaks in a group of lines thus eliminating spurious fluctuations coming from the different resolution between the synchrotron radiation and $\mathrm{He} \mathrm{I}$ spectra. Furthermore, the peaks $\mathrm{H}_{2} \mathrm{O}^{+}, \mathrm{OH}^{+}$ and $\mathrm{H}^{+}$were also removed from analysis since we clearly detect signs of a small water contribution. This contribution changed in the He I and synchrotron radiation spectra. From this graph we can observe that the ratio between the partial productions of the two spectra (the one with the discharge lamp and the one with synchrotron radiation) increases for heavier masses. This could support our hypothesis of mass discrimination stated above.

In summary, time-of-flight mass spectrometry has been employed to the study of the natural amino acid DL-proline using synchrotron radiation as the ionization source. Total ion yield and mass spectra were recorded from 13 to $21.6 \mathrm{eV}$, using a metallic/gas filter coupled to the beamline in order to reduce the contribution from higher order harmonics contamination. Fragmentation products were identified and the relative production among them for a few photon energies was obtained. The loss of the $\mathrm{COOH}$ fragment represents the most probable dissociation pathway following the photoionization of DL-proline in the valence region.

\section{ACKNOWLEDGMENTS}

The authors wish to acknowledge the staff of the LNLS and support from the Brazilian agencies CAPES, CNPq, FAPERJ, FAP-DF and FAPESP.
[1] A. Brack (Ed.), The Molecular Origins of Life, Cambridge University Press, UK (1998).

[2] L. E. Snyder, Origins Life Evol. Biosphere 27, 115 (1997).

[3] M. H. Engel and B. Nagy, Nature (London) 296, 837 (1982).

[4] I. Powis, E. E. Rennie, U. Hergenhahn, O. Kugeler, and R. Bussy-Socrate, J. Phys. Chem. A 107, 25 (2003).

[5] H.-W. Jochims, M. Schwell, J-L. Chotin, M. Clemino, F. Dulieu, H. Baumgartel, and S. Leach, Chem. Phys. 298, 279
(2004).

[6] A. F. Lago, L. H. Coutinho, R. R. T. Marinho, A. Naves de Brito, and G. G. B. de Souza, Chem. Phys. 307, 9 (2004).

[7] NIST, Chemistry webbook (http://webbook.nist.gov/chemistry).

[8] F. Burmeister, L. H. Coutinho, R. R. T. Marinho, M. G. P. Homem, M. A. A. de Morais, A. Mocellin, O. Bjorneholm, S. L. Sorensen, P. T. Fonseca, J. G. Pacheco, and A. Naves de Brito. Submitted to J. Elect. Spect. Rel. Phen. (2004). 
[9] A. Mocellin. R. R. T. Marinho, L. H. Coutinho, F. Burmeister, K. Wiesner, and A. Naves de Brito, Chem. Phys. 289, 163 (2003).

[10] P. T. Fonseca, J. G. Pacheco, E. D. Samogin, and A. R. B. de Castro, Rev. Sci. Instrum. 63, 1256 (1992).
[11] R. L. Cavasso-Filho, M. G. P. Homem, R. Landers, and A. Naves de Brito. J. Elect. Spect. Rel. Phen. 144-147, 1125 (2005). 